\title{
Reutilização de informações no projeto de moldes de injeção através do uso de modelos de informação e de conhecimento
}

\author{
Carlos Alberto Costa, Ph.D. \\ Universidade de Caxias do Sul \\ Departamento de Engenharia Mecânica \\ Caxias do Sul-C.P. 1352 - 95001-970 \\ Rio Grande do Sul - cacosta@ucs.br \\ Robert I. M. Young, Ph.D. \\ Loughborough University \\ Department of Manufacturing Engineering \\ Loughborough - Leicestershire - LE11 3TU \\ England-UK, $\underline{\text { Rim@lboro.ac.uk }}$
}

\begin{abstract}
O uso de modelos de informações tem sido reconhecido como uma abordagem bastante eficiente para apoio às fases de desenvolvimento de produtos. Tradicionalmente este uso tem se limitado aos modelos de produtos, com ênfase nos seus aspectos geométricos. Este artigo estende o conceito tradicional de modelos de informações, para modelos adicionais de informações e de conhecimentos, visando apoiar as decisões durante as fases iniciais de projeto do produto. O conceito de um modelo adicional de informações e de conhecimento, chamado Modelo de Produto Variante, é introduzido e discutido. Este modelo visa apoiar as atividades de projeto variante através da reutilização de informações e de conhecimento. A estrutura deste modelo é apresentada e discutida em termos das funções do produto e suas respectivas soluções de projeto, bem como as interações existentes entre estas soluções, que são avaliadas em relação às especificações do produto e restrições de projeto. Para tanto, a relação entre o Modelo de Produto Variante e modelo do produto são definidas através de elementos chamados Links de Conhecimento. $\mathrm{O}$ trabalho utilizou o ambiente de projeto de moldes de injeção como caso para explorar as idéias apresentadas e a implantação de um sistema de apoio à tomada de decisões.
\end{abstract}

Palavras-chave: modelos de informações; projeto variante; moldes de injeção; reutilização de informações; modelo de produto variante.

The application of information models, such as product model, has been recognised as an important approach to support product development life-cycle phases. In this paper such approach is extended to support information and knowledge reuse during the product design initial stages. The concept of a new information model, called Product Range Model, is presented and discussed. It is applied to support variant and adaptive design activities through the information and knowledge reuse. Its structure is presented in terms of product functions and their respective design solutions, and how the interactions among these design solutions can be evaluated against the product specifications and design requirements. The relationships between the product model and the Product Range Model are maintained through the concept of knowledge-links elements, which are also part of the Product Range Model structure. In order to explore the ideas presented in this paper, injection mould tooling has been used as an appropriate product range and evaluated through the design and implementation of a design support system utilising object-oriented database.

Keywords: information models; variant design; injection mould; information reuse; product range model. 


\section{Introdução}

O processo de desenvolvimento de um produto é caracterizado por uma sequiência de etapas diferentes que objetivam interesses inerentes a cada uma delas como, por exemplo, estética, funcionalidade, fabricação e montagem do produto final. Um dos grandes desafios das empresas que tentam disputar e se manter no mercado atualmente é o de cumprir tais etapas num menor tempo e com a certeza que seus produtos serão concebidos dentro dos melhores critérios de qualidade e custos.

Filosofias e técnicas como Engenharia Concorrente (CE) têm propiciado formas mais inteligentes, integradas e otimizadas de se lidar com as informações envolvidas no desenvolvimento de novos produtos (JO, PARSAEI e SULLIVAN, 1993). Dentro destas, uma das abordagens utilizadas tem sido a aplicação de modelos de informações.

A aplicação de modelos de informação, como por exemplo, modelo de produto, tem ganhado bastante atenção da comunidade industrial nos últimos anos (ANDERL, 1997). A definição de uma representação de estrutura de dados integrada para compartilhar e trocar dados é definida também na ISO 10303-STEP, particularmente na descrição geométrica de um produto (GU e CHAN, 1995). A definição desta estrutura é crucial para permitir que aplicativos computacionais possam compartilhar e armazenar dados no modelo do produto (McKAY, BLOOR et al., 1996).

Algumas pesquisas, um pouco mais recentes, vêm explorando formas de capturar de uma forma inteligente não só aspectos geométricos, mas também possibilidades de variações na configuração de produtos do tipo variante (ERENS e VERHULST, 1997) sendo que iniciativas como a da ISO (AP214), começam a reconhecer e melhorar a representação para a captura destes tipos de produtos através do conceito de "classes de produtos" (MANNISTO et al., 1998). Tais iniciativas têm resultado em um avanço na representação da estrutura do produto sob uma perspectiva funcional do mesmo, o que pode fornecer uma melhor compreensão de sua arquitetura.

Para fornecer um suporte mais efetivo às diferentes fases do projeto de um produto, um modelo de informações deve capturar não somente informações puras, mas o conhecimento adquirido ao longo do tempo e que é relevante para aquele produto. Isso deveria incluir as relações entre as funções, suas necessidades e seus significados (SIVALOGANATHAN e SHAHIN, 1999). Esta captura torna-se ainda mais útil no caso de produtos que possuem um projeto do tipo variante, onde a reutilização de informações e conhecimento sobre o produto são mais intensas. O conceito de produto do tipo variante neste trabalho está relacionado a tipos de produtos onde o seu conceito de projeto já está bem definido, mas as alternativas de como este conceito pode ser incorporado e combinado, para cada novo caso, poderá resultar em novos projetos, ou soluções.

Assim, outros modelos de informações, também chamados de modelos adicionais de informações e conhecimento, têm sido reconhecidos como necessários para o apoio às atividades integradas dentro do ciclo de desenvolvimento de produtos. Como exemplo pode-se citar o modelo de manufatura (MOLINA $e t$ al., 1995) que apoia as decisões relacionadas ao projeto para a manufatura, e o Modelo de Produto Variante, proposto por este trabalho, que visa apoiar a reutilização de informações e conhecimentos relacionados às fases iniciais de projeto de um produto. A Figura 1 mostra a representação de uma arquitetura geral de um ambiente baseado na abordagem de modelos de informação.

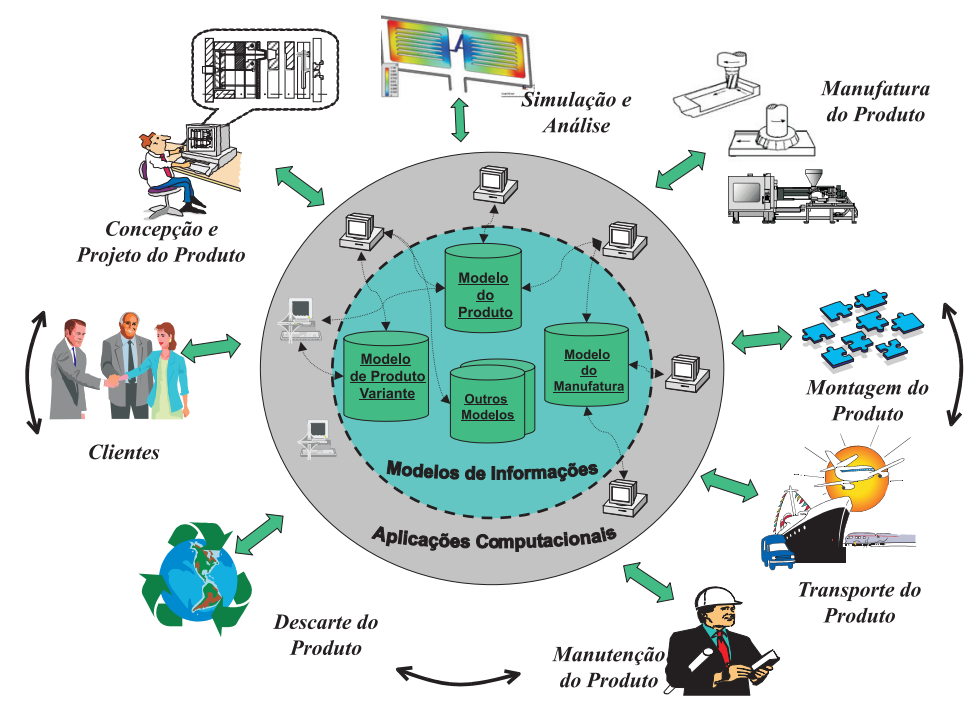

Figura 1 - Arquitetura geral de um ambiente baseado em modelos de informações. 
Nesta abordagem os modelos de informações capturam como e onde as informações relacionadas a um produto são armazenadas e como as mesmas se tornam disponíveis para as diferentes aplicações computacionais durante o ciclo de projeto e a fabricação de tal produto. Para permitir o uso dos modelos de informações, uma estrutura de dados deve ser definida para sua representação, uma vez que baseadas nelas as aplicações computacionais irão armazenar ou recuperar as informações. Os modelos de informações estão, normalmente, relacionados com a aplicação de tecnologias relacionadas com banco de dados.

Assim, cada banco de dados fornece acesso a tipos particulares de informações, i.e., o modelo de produto oferece informações sobre o produto, o modelo de manufatura oferece informações relacionadas às facilidades de manufatura e o Modelo de Produto Variante oferece informações e conhecimento para apoiar decisões de novos projetos baseado em projetos anteriores. Este artigo diferencia informação de conhecimento no sentido que informação é um dado estruturado que possui algum significado, e o conhecimento é a informação com um valor adicionado que relaciona como ela pode ser aplicada ou utilizada. $\mathrm{O}$ modelo de produto, que apoia um compartilhamento integrado de informação, é um modelo de informações, enquanto o Modelo de Produto Variante trata-se de um modelo de informações e conhecimento (COSTA e YOUNG, 2001).

Ainda com relação à Figura 1, as aplicações computacionais focalizam a captura e representação da funcionalidade específica de uma determinada atividade de projeto ou fabricação (p.ex. Projeto para a Manufatura), além de manter uma interface com o usuário final (projetista). Tal funcionalidade poderá estar baseada puramente em leis físicas ou em procedimentos adotados dentro de uma empresa específica em função de uma estratégia maior. As aplicações computacionais são responsáveis também pelas mudanças (recuperação ou armazenamento) dos dados nos modelos de informações e normalmente estão relacionadas com ferramentas computacionais, tais como computação gráfica, sistemas especialistas, sistemas baseados em conhecimento etc.

O uso destes dois elementos, modelos de informações e aplicações computacionais, em harmonia, fornece às empresas algumas vantagens tais como: uma completa integridade nos dados, rápida flexibilidade, manutenção, independência de "vendedores" e suporte para o ciclo de vida do produto.

Dentro da abordagem adotada neste trabalho, o Modelo de Produto Variante fornece um repositório de informações e de conhecimentos separado do modelo de produto, que pode ser utilizado para armazenar as formas pelas quais um produto variante pode, ou não, ser projetado. Para poder melhor guiar o projetista, as funções deste tipo de produto são associadas com as possíveis soluções de projetos. O conhecimento para apoiar a reutilização inteligente dos conceitos de projeto é capturado através de elementos chamados de "interações". A idéia principal do Modelo de Produto Variante é oferecer ao projetista informações de qualidade que permitam ao mesmo tomar as decisões mais apropriadas. Esta abordagem não impõe um processo ou uma sequiência específica de projeto para o projetista, mantendo o princípio de que a estrutura de modelo de produto pode ser utilizada como repositório das informações sobre o produto em desenvolvimento.

O trabalho apresentado neste artigo adotou uma abordagem baseada em orientação a objetos para modelar a estrutura do modelo de informação definido. $\mathrm{O}$ banco de dados orientado a objetos ObjectStore foi utilizado para implementar a estrutura de informações, em combinação com o Visual C++ 5.0, para realizar a aplicação funcional do software. Para explorar as idéias envolvidas no conceito apresentado, moldes de injeção foram escolhidos como produtos de projeto do tipo variante.

\section{0 ambiente de projetos de moldes de injeção}

\subsection{Desafios no projeto de moldes de injeção}

O ciclo produtivo de produtos plásticos injetados é composto por diferentes etapas multidisciplinares que passam pelo projeto do componente plástico, projeto do molde, fabricação do molde e, finalmente, a injeção da peça plástica. Apesar da maior parte dos custos estarem envolvidos com a fabricação do molde e injeção do componente plástico, as fases de projeto são determinantes para o sucesso destes produtos/custos. É reconhecido que $60 \%$ dos custos de fabricação de um produto são decididos já na fase de projetos (HSU e WOON, 1998). Isto traz para as atividades relacionadas com o projeto do molde uma importância ainda mais acentuada dentro do ciclo produtivo de injeção de plásticos, seja pela concorrência cada vez mais acentuada entre empresas e rapidez em definir uma correta e rápida cotação, seja pela complexidade natural das áreas de conhecimento envolvidas no projeto do molde. Dentro deste cenário, uma questão que tem surgido nos últimos anos é: como conciliar um ambiente concorrente com o uso de informações que podem realmente suportar o projeto e fabricação de moldes? A resposta não é muito simples e ainda é razão de muitas pesquisas e anseios, seja no ambiente acadêmico, seja no ambiente industrial (COSTA e YOUNG, 1999; CHIN e WONG, 1996; LEE et al., 1997). 
Dois tópicos bastante discutidos nos últimos anos relacionados com os sistemas integrados para o apoio ao ciclo de desenvolvimento de produtos apontam para:

- a importância de se dispor de modelos de informações comuns integrados e compartilhados entre as diferentes aplicações computacionais dentro da empresa e

- a importância da captura, representação e disponibilização de informações e conhecimento ao longo do tempo dentro de uma empresa.

Neste contexto, os sistemas modernos de apoio ao projeto de moldes de injeção deverão estar preparados para além de capturar as informações e conhecimento envolvidos no processo, disponibilizar tais informações para reutilização futura com qualidade, para o apoio às decisões futuras.

\subsection{O molde de injeção como produto do tipo variante}

A idéia básica, ou conceito, que define um molde de injeção é bem entendida, embora possam haver diferentes variações no projeto final de um molde, dependo de como o projetista julgar que deve atender aos requisitos de projeto, dentro de suas condições. Além dos aspectos geométricos relacionados com a impressão do componente plástico (o resultado da junção de cavidade e macho), o molde possui também especificações básicas como número de impressões e o tipo de construção do molde, e.g., duas ou três placas. Existem também outras necessidades de projetos que devem ser consideradas e que estão relacionadas com a alimentação, refrigeração, extração etc. do molde. Tais necessidades podem ser atendidas por diferentes conjuntos de soluções de projeto, onde cada um destes visa satisfazer funções básicas do produto molde de injeção, tais como ejetar a peça plástica, alimentar o molde, refrigerar a cavidade etc. (ROSATO e ROSATO, 1995). A Figura 2 mostra o caso dos moldes de injeção onde pode ser identificado um grupo definido de funções, bem como suas respectivas soluções e suas relações, o que pode orientar o projetista em suas fases iniciais de projeto (MENGES e MOHREN, 1993).

Este trabalho usa como base de estudo este conjunto de funções e suas respectivas "possíveis" soluções (chamadas aqui de soluções de projeto) para atender as condições de projeto. Estas soluções são ditas "possíveis" pois a escolha de uma específica dependerá dos requisitos e restrições de projeto para cada molde de injeção. Um requisito pode ser o molde ter uma vida útil pequena, por exemplo 1000 peças, enquanto uma restrição pode ser em função da própria geometria da peça a ser injetada. Assim, apesar destas relações entre funções e suas "possíveis" soluções estarem bem definidas, pouca utilidade as mesmas teriam caso não existissem critérios definidos para suas aplicações, fazendo assim que a escolha do melhor conjunto de soluções não seja tão simples assim.

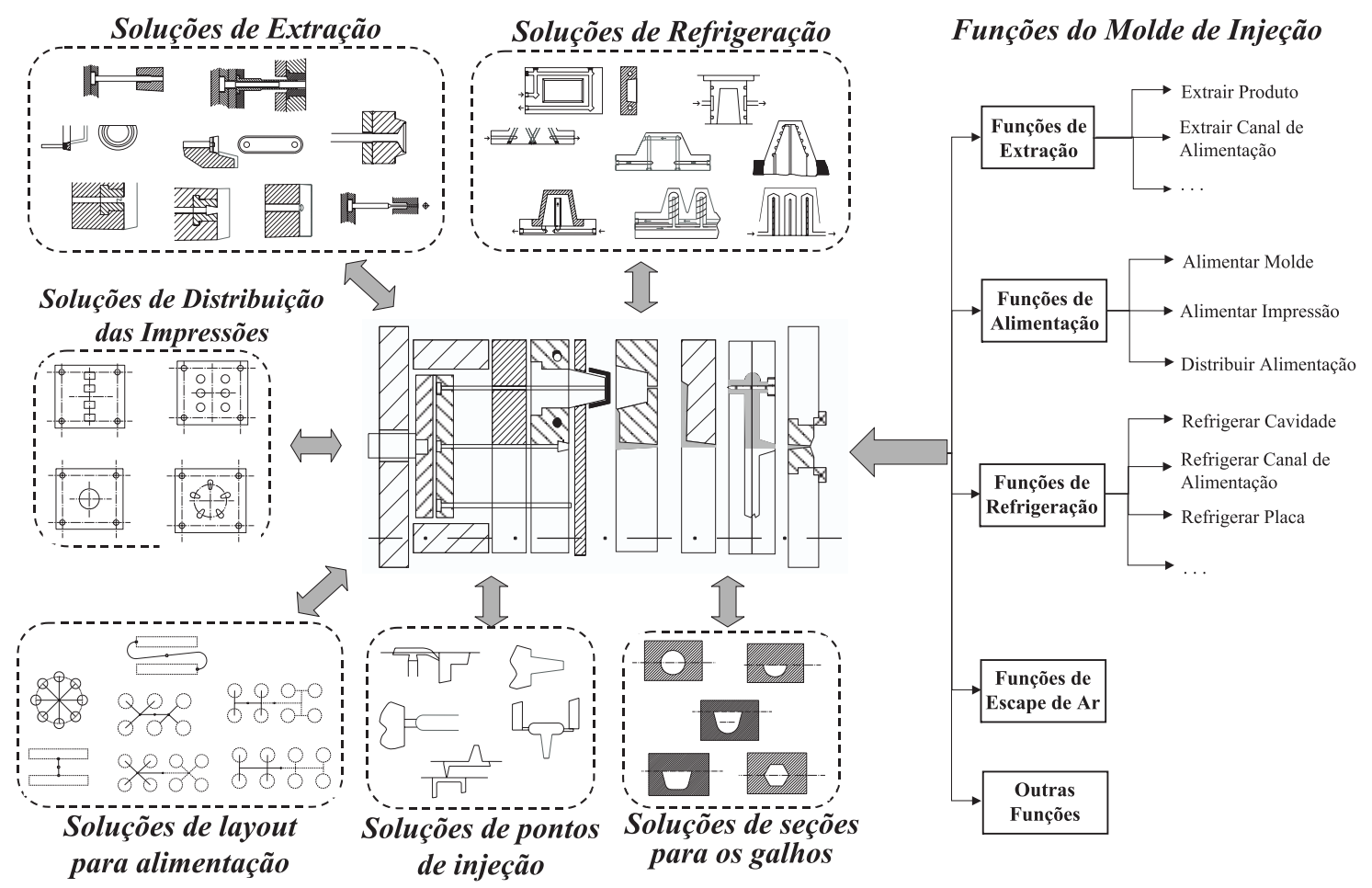

Figura 2 - Exemplo de funções e soluções para moldes de injeção 
O grande desafio para os projetistas menos experientes, durante as fases iniciais de um novo projeto de um molde de injeção, está relacionado com a decisão ou escolha do melhor conjunto de tipos de técnicas, ou soluções, que serão utilizadas para atender a cada uma das funções, ou necessidades, de projeto do mesmo. Isto se deve ao grande número de variáveis e interações existentes entre elas, como por exemplo, especificações sobre a peça plástica, configuração básica do molde e do sistema de alimentação etc. Somado a isto, existem ainda outras interações em função das decisões de projeto, como por exemplo, as interações existentes entre os sistemas de extração, refrigeração e/ou alimentação, como representado através da Figura 3.

Assim, o gerenciamento destas interações durante o processo de projetar o molde de injeção é fundamental para um projeto final balanceado. Estas interações e a forma de priorizá-las durante o projeto do molde é que fazem parte do conhecimento adquirido ao longo do tempo pelos projetistas mais experientes, ou pela empresa, devendo estes ser capturados de alguma forma. Este trabalho explora como estas interações deveriam ser capturadas, em conjunto com as funções e soluções de projeto, para o apoio ao projeto de moldes.

Diferentes pesquisas têm sido conduzidas no sentido de se automatizar e otimizar o processo de projetar moldes de injeção. Contudo, a maior parte tem se especializado em sistemas específicos do molde (ONG et al., 1995; IRANI et al., 1995; WANG et al., 1996). Desta forma, uma das questões que ainda permanece aberta é como gerenciar as interações entre diferentes decisões de projetos no caso de moldes de injeção, sendo esta uma área onde este trabalho pretende contribuir.

\section{Modelo de Produto variante apoi- ando a reutilização de informações no projeto de moldes de injeção}

O Modelo de Produto Variante é um modelo de informações que armazena e compartilha não somente as relações entre funções e soluções de projeto mas também, os critérios de projeto que devem ser atendidos para a aplicação de cada solução de projeto. Estes critérios são definidos através das interações que existem entre cada uma destas possíveis soluções e as especificações iniciais e outras decisões de projeto feitas pelo projetista. Para a definição da estrutura deste modelo, alguns aspectos foram explorados e são discutidos nas próximas seções.

\subsection{Funções e soluções de projeto}

A Figura 4 mostra uma representação geral de algumas das funções principais que devem ser atendidas em um molde de injeção e algumas de suas respectivas soluções de projeto. Apesar da descrição das principais funções de um molde de injeção e seus sistemas poder ser encontrada na literatura (ROSATO e ROSATO, 1995; MENGES e MOHEN, 1993), poucos trabalhos têm definido explicitamente as suas relações. Neste trabalho tais relações são definidas e utilizadas como base para explorar como os critérios de projeto associados a cada solução de projeto podem fornecer opções de escolha para o projetista.

Assim, cada função estará associada a um conjunto de todas as possíveis soluções de projeto que possam atendê-la. Como as funções de um molde podem ser também decompostas em sub-funções mais específi-

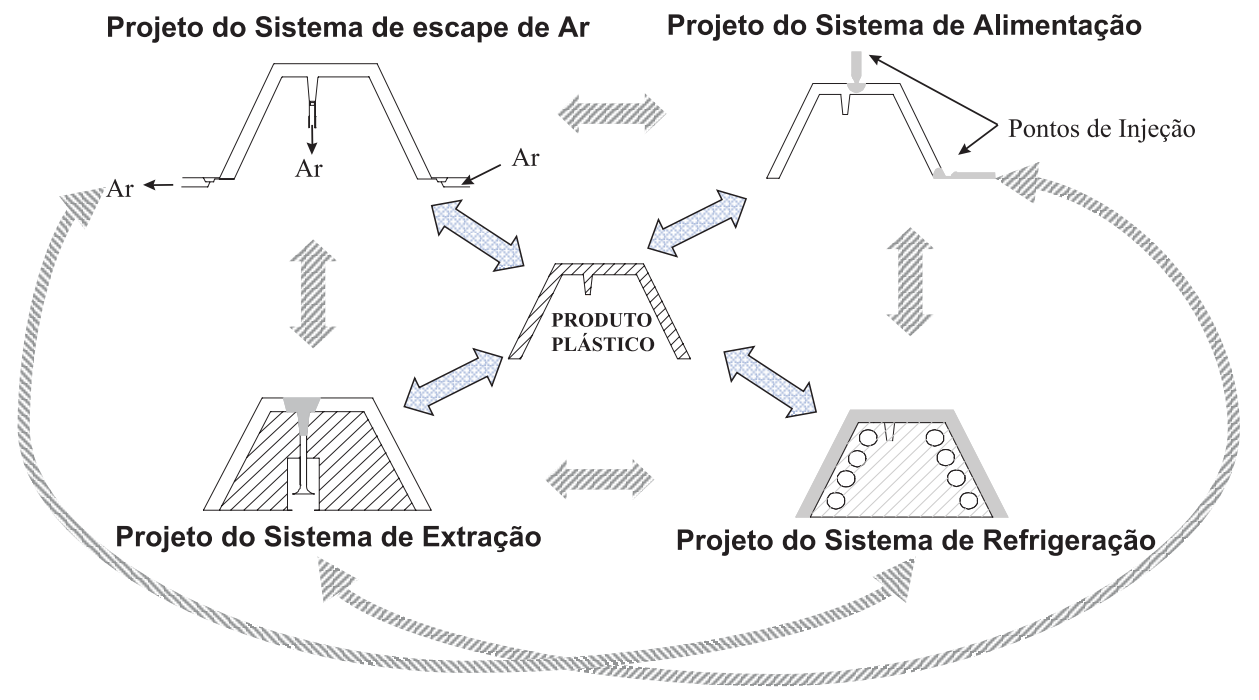

Figura 3 - Exemplo de interações no projeto do molde de injeção 


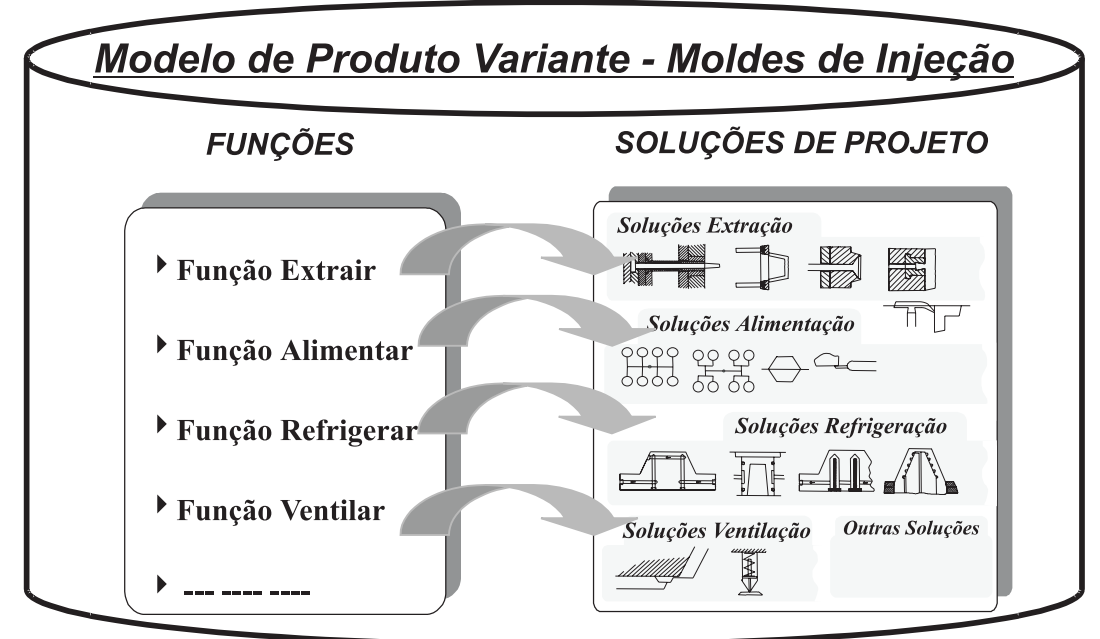

Figura 4 - Exemplo das relações gerais entre funções e soluções para moldes de injeção

cas, e.g., refrigerar molde pode ser decomposta em refrigerar cavidade, refrigerar macho, refrigerar canal de alimentação etc., o grupo de soluções de projeto inicial passa a ser mais específico para cada uma destas sub-funções.

O processo de pesquisa pelas soluções válidas de projeto passa inicialmente por uma pesquisa de seleção de todas as soluções associadas com a função escolhida. O conjunto de soluções selecionadas é, então, verificado com relação às condições de projeto, i.e., especificações, necessidades e outras decisões já tomadas pelo projetista. Somente as soluções válidas deverão ser ofertadas ao projetista.

\subsection{Interações entre as soluções de projeto}

Uma simples relação entre as funções e suas possíveis soluções de projeto não trazem uma informação muito útil ao projetista. Para ser oferecida como uma opção válida de projeto, cada solução deverá respeitar todos os critérios de projeto previamente estabelecidos. Estes critérios poderão estar associados com diversos tipos de informações, como por exemplo: número de cavidades do molde, tipo de sistema de alimentação, propriedades e características da peça plástica, tipos de técnicas escolhidas para o sistema de extração, galho, ponto de injeção e refrigeração. Para tanto, é necessário a captura do conhecimento que representa estes critérios de projeto.

Na pesquisa em questão, foram definidos elementos chamados "interações" que são responsáveis por esta captura. O conjunto destes elementos, combinados dentro de uma lógica, é que permitirá avaliar quando uma solução de projeto pode ser considerada válida, ou não válida, para uma determinada situação/ especificação de projeto.
A Figura 5 mostra um exemplo de um conjunto de interações associado com uma solução de projeto relativa ao sistema de alimentação do molde, i.e. injeção submarina. Para que tal solução possa ser considerada válida, a mesma deverá respeitar os critérios definidos em relação à configuração do molde, por exemplo 2 placas, linha de fechamento junto à superfície de fechamento e outros tipos de interações. Estas interações poderão ser oriundas de regras definidas na literatura ou baseadas no conhecimento (heurística ou melhores práticas) de projetistas mais experientes ou estratégias da empresa. Também, deverão estar associadas a cada uma das soluções potenciais de projeto para atender a uma função específica do molde de injeção.

Para cada elemento de interação foram identificados três aspectos relacionados com suas informações, que são:

- a localização da informação, que é relativa à localização de uma informação específica que será comparada com a informação armazenada no elemento de interação. Neste sentido as informações armazenadas no modelo de produto devem ser identificadas e a estrutura de dados que representa este modelo deve ser conhecida, fornecendo o caminho correto para sua recuperação;

- o tipo de informação, que está relacionado com o tipo específico da informação que será comparada. Neste sentido dois tipos de informações foram inicialmente identificados: numérica (relacionada com atributos numéricos), e.g., número de cavidades; e de existência (que está relacionada com a existência, ou não, de uma característica particular do produto). Este aspecto é que garante a compatibilidade entre as informações que estão sendo comparadas, e 


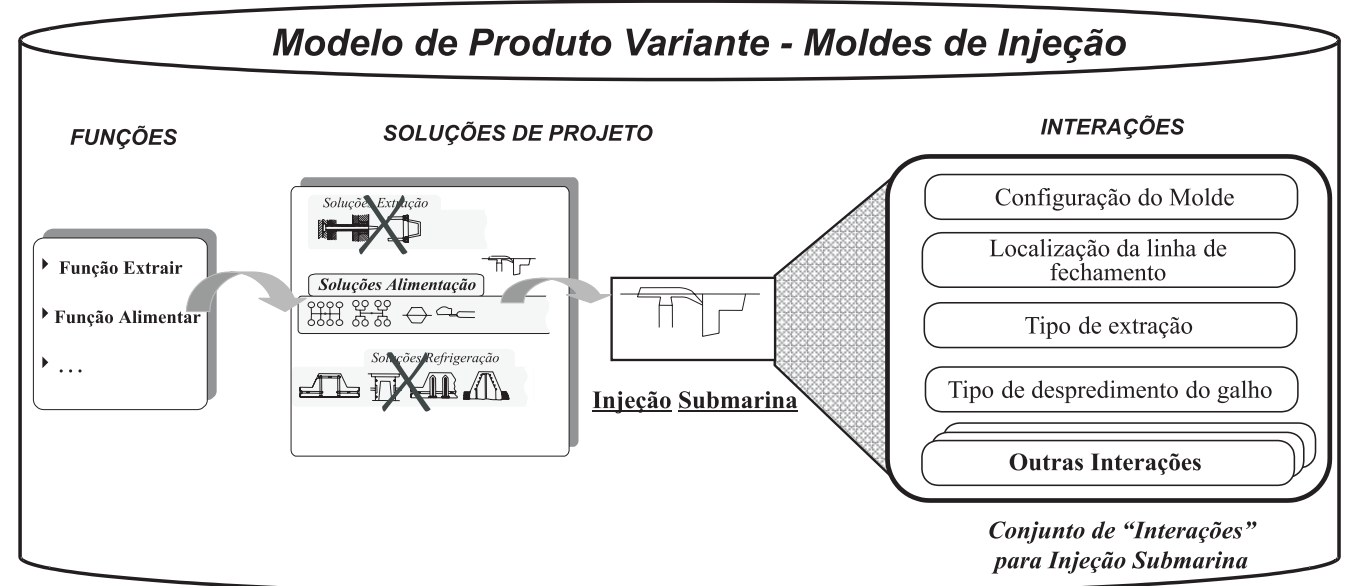

Figura 5 - Exemplo de um conjunto de interações para uma solução de projeto.

- a importância da informação, que define em que nível a interação pode, ou não, restringir uma solução de projeto. Assim as interação podem ser definidas como necessárias, desejáveis etc.

\section{Relações entre os modelos de informações}

Dentro da abordagem usada por este trabalho, os modelos de informações utilizados, i.e., modelo do produto e Modelo de Produto Variante, estão definidos de formas separadas (COSTA e YOUNG, 2001). Isto propicia uma melhor preservação das informações e conhecimento armazenados em ambos os modelos ao longo do tempo, permitindo uma evolução de cada um interdependentemente.

Assim, o Modelo de Produto Variante precisará acessar as informações do produto em projeto, para poder avaliar a validade de soluções de projeto potenciais em uma determinada situação. Para permitir o relacionamento entre as estruturas de dados destes dois modelos foram criados elementos, chamados Links de Conhecimento (knowledge links), que viabilizam a recuperação das informações do produto. Estes elementos, que são associados a cada uma das interações, guardam o caminho de onde uma informação específica deve ser recuperada no modelo do produto. Esta informação poderá ser um atributo de um objeto ou o próprio objeto. A Figura 6 mostra um exemplo de como as interações podem ser avaliadas através dos Links de Conhecimento.

Para cada interação associada com uma solução de projeto, um atributo específico é pesquisado e recuperado do Modelo de Produto e então comparada com o valor de referência que esta interação armazena. Por exemplo, nesta situação, para que a injeção submarina pudesse ser utilizada a configuração do molde deverá

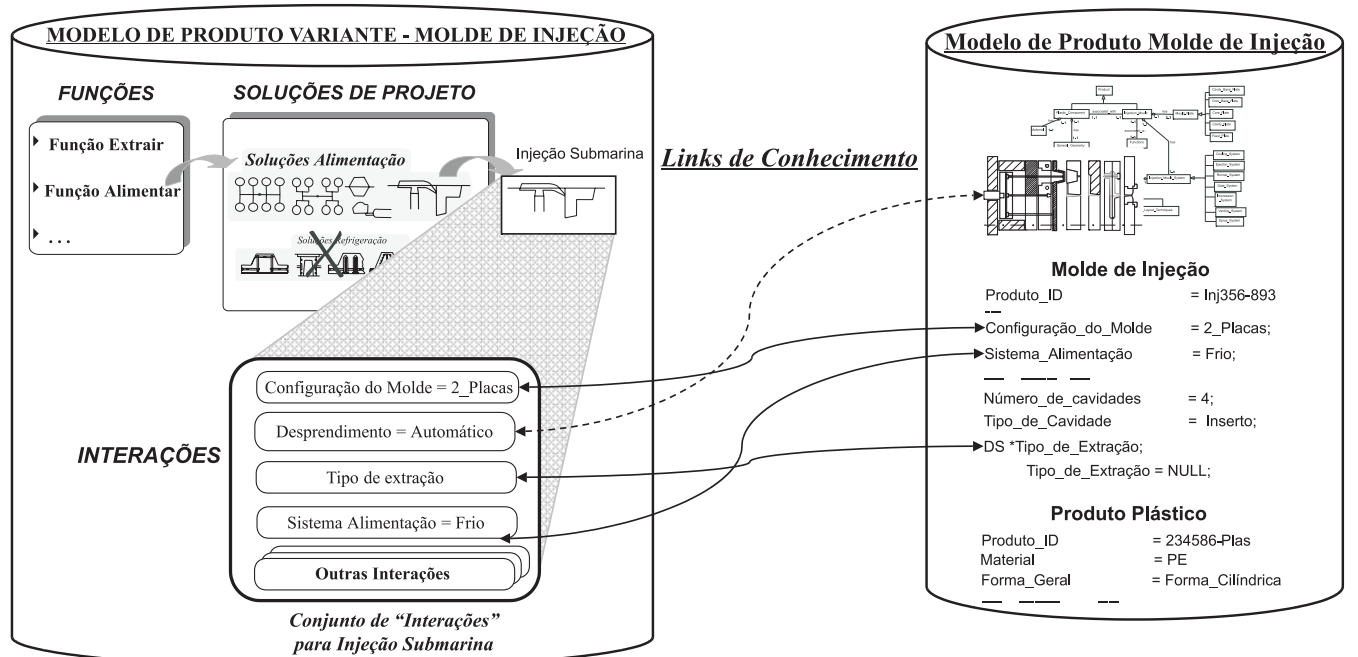

Figura 6 - Exemplo de relacionamento entre os modelos de informações através dos Links de Conhecimento. 
ser do tipo 2_placas e um sistema de alimentação que não poderá ser do tipo câmara quente.

\section{Definição das estruturas para os modelos de informações}

Para o processo de análise e projeto do sistema de reutilização de informações, foi utilizada aplicação da notação UML (Unified Modelling Language) dentro do modelo de referência RM-ODP (Reference Model for Open Distributed Processing) [COSTA et al, 2001]. A aplicação desta notação permitiu a análise e projeto do sistema tanto em termos de funcionalidade básica para apoiar o projeto de moldes de injeção, como para definição das estruturas de informações a serem utilizadas pelo mesmo.

A Figura 7 e a Figura 8 mostram as representações gerais das estruturas de informações que representam o modelo de produto e Modelo de Produto Variante para o caso de moldes de injeção.

Enquanto o Modelo do Produto está direcionado à captura de uma estrutura que permita uma melhor representação dos produtos molde de injeção e peça plástica, o Modelo de Produto Variante está direcionado à captura das informações e relações específicas que representam o conhecimento para a escolha de melhores opções de projeto.

No caso da Figura 7, as informações sobre dois tipos de produtos podem ser armazenadas, i.e. Componente
Plástico (Plastic_Component) e Molde de Injeção (Injection_Mould). Esta última apresenta relações com as classes Placas do Molde (Mould_Plate) e Sistemas do Molde (Injection_Mould_System), que podem ser do tipo refrigeração (Cooling_System), extração (Ejection_System), alimentação

(Runner_System) etc. Cada Sistema do Molde poderá ainda ter uma ou mais Soluções Técnicas (Solution_Techniques) de projeto para atender a funcionalidade de seu sistema. Por exemplo, para um sistema de extração de um molde poderão ser utilizadas técnicas de extração por pinos, anéis e ar. Os Sistemas do Molde poderão ainda possuir relações entre eles como, por exemplo, um sistema de refrigeração para sistemas de extração.

Na Figura 8 é representado como cada uma das Funções (Functions) definidas para moldes de injeção, e.g. extrair peça, refrigerar macho etc., podem estar associadas com diferentes Soluções de Projeto (Design_Solutions), e.g. soluções de extração, soluções de refrigeração etc. As funções podem ser decompostas em outras (sub)funções que estarão associadas a conjuntos de Soluções de Projeto mais específicas.

A estrutura da classe Soluções de Projeto (Design_Solutions), em seu nível mais básico, é compatível com a estrutura da classe Soluções Técnicas (Solution_Techniques) do modelo de produto. Isto permite que soluções específicas selecionadas no Modelo de Produto Variante sejam armazenadas no modelo de produto.

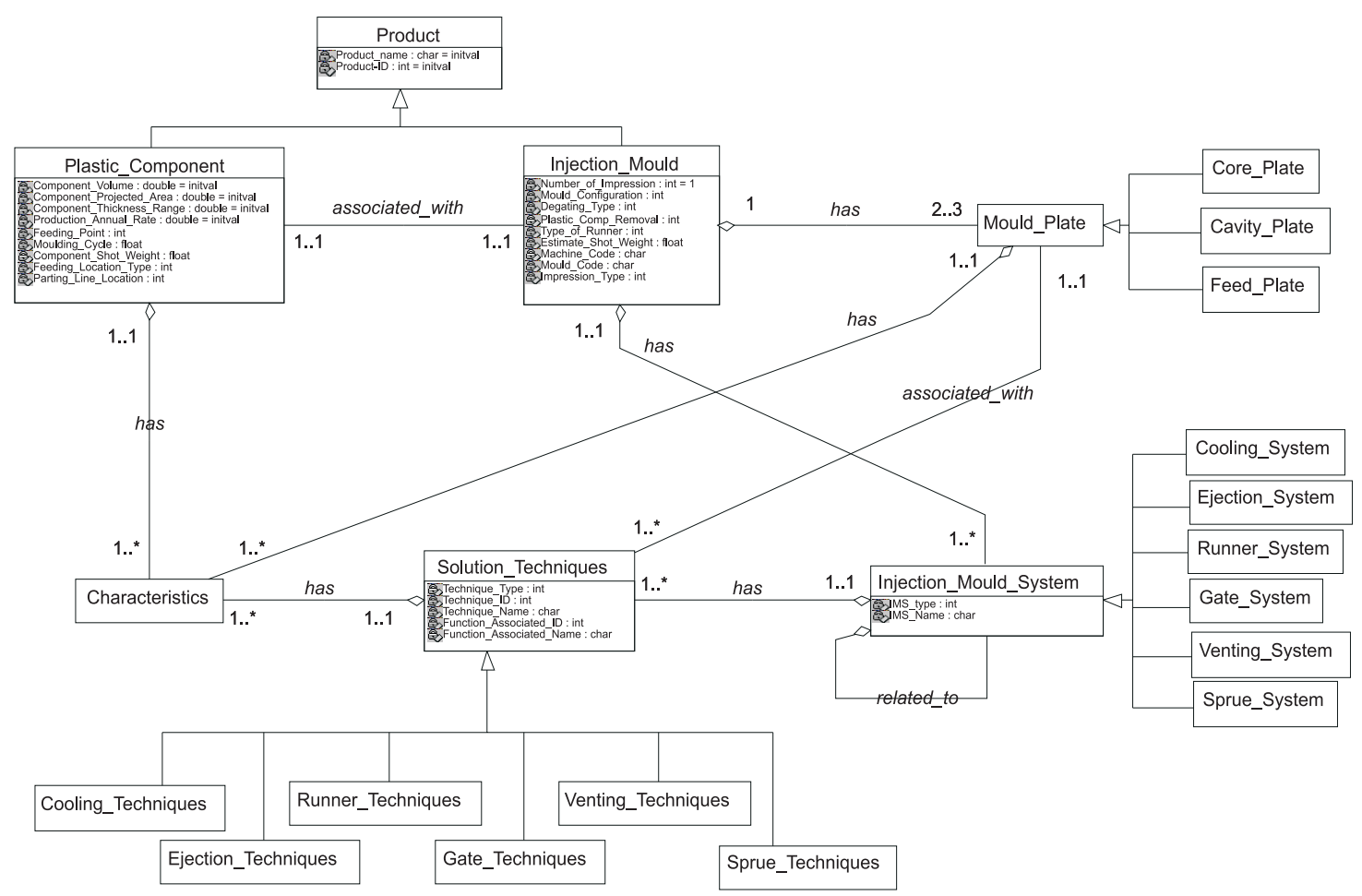

Figura 7 - Representação UML do modelo de produto para moldes de injeção. 
A classe Soluções de Projeto está associada com a classe Opções de Fabricação

(Manufacturing_Options) que abrange as possíveis formas de se fabricar e/ou comprar (elementospadrão) uma solução.

Um conjunto de Interações (Interactions) define o critério de projeto a ser empregado para a aplicação de cada Solução de Projeto. Dois tipos de interações foram definidos neste trabalho: Interações Simples (Simple_Interactions) e Interações Compostas (Composite_Interactions), podendo esta última ser composta de outras interações, Simples ou Compostas, dentro de uma ordem booleana determinada. As Interações Simples é que possuirão um relacionamento com o modelo de produto. Através das Associações de Conhecimento (Knowledge_Links), guardam o caminho para que um determinado atributo seja recuperado no Modelo de Produto.
Para a captura da funcionalidade associada ao uso das informações representadas no modelo de produto e Modelo de Produto Variante, foram utilizados diagramas de sequência da UML. A Figura 9 mostra a funcionalidade básica definida dentro do sistema de reutilização de informações, através dos métodos que são chamados durante o processo de verificação por soluções de projeto válidas. Após a seleção de uma função específica pelo usuário, um conjunto de soluções de projeto que podem ser aplicadas a esta função, é selecionado. Cada solução de projeto tem suas interações avaliadas, retornando sua condição: aprovada ou reprovada. Esta comparação é feita com as informações recuperadas do modelo de produto, através das Associações de Conhecimento. Com base na condição de cada elemento de interação, as soluções de projeto são avaliadas, retornando ao usuário com a condição de válida ou rejeitada.

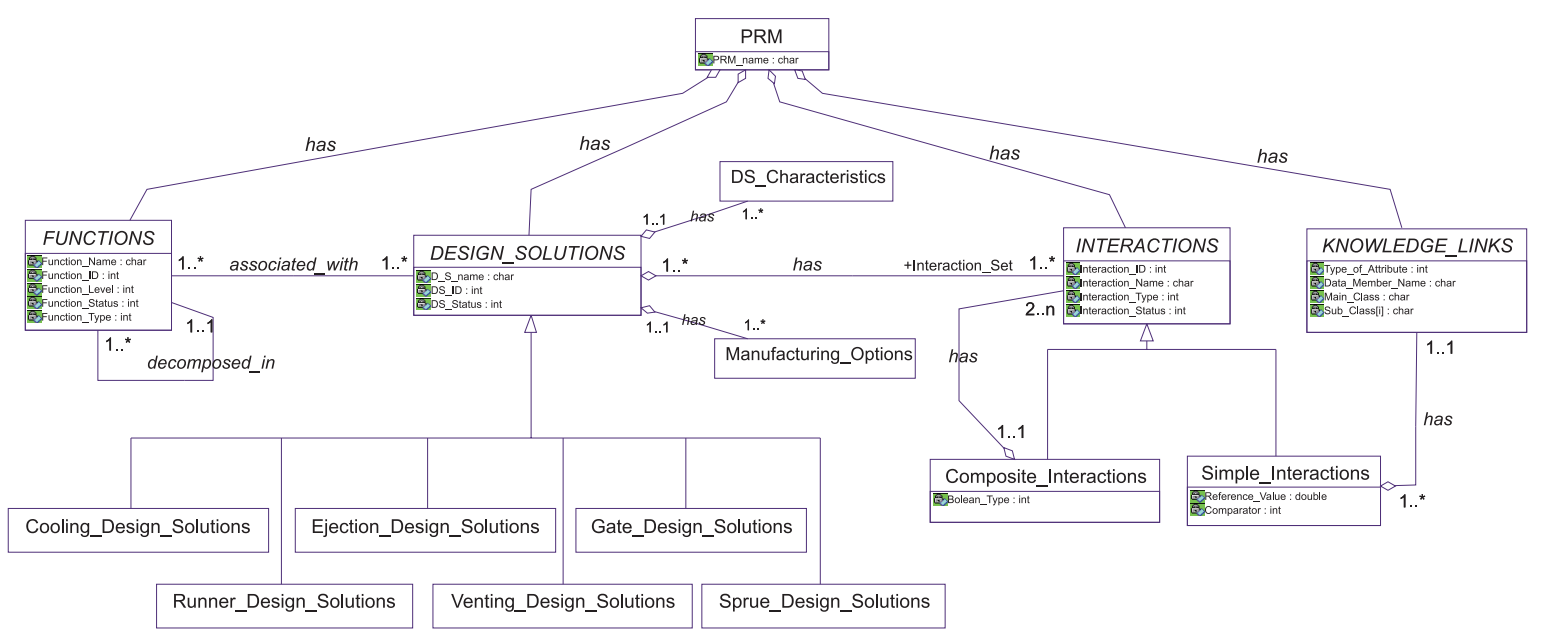

Figura 8 - Representação UML para o Modelo de Produto Variante para moldes de injeção.

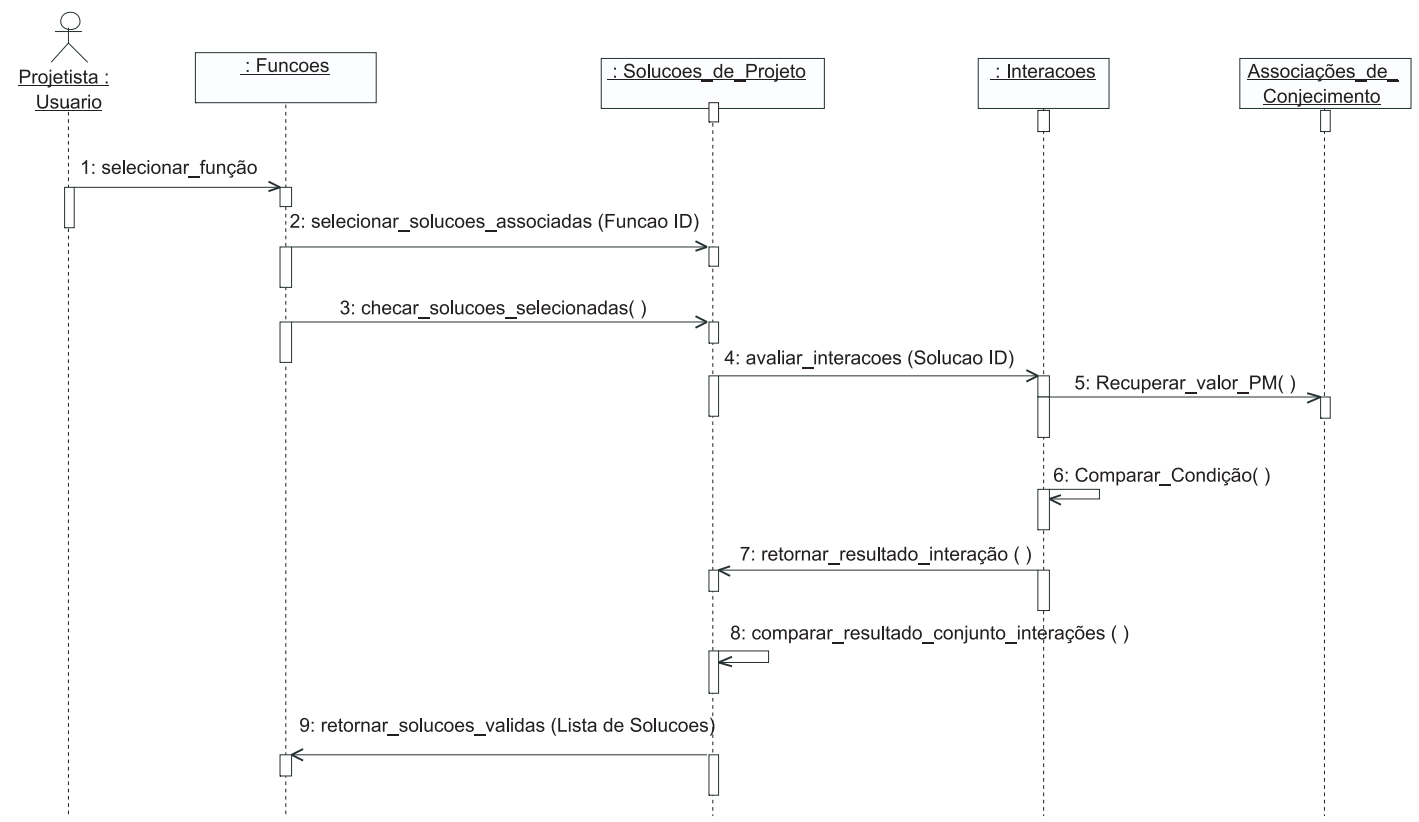

Figura 9 - Relações funcionais entre as classes do Modelo de Produto Variante. 


\section{Sistema Experimental de Reutilização de Informações no Projeto de Moldes de Injeção}

Para a implementação computacional do sistema proposto foram utilizados um banco de dados orientado a objetos (ObjectStore $\left.{ }^{\circledR}\right)$ e um ambiente de programação (Visual C++®). Enquanto o ObjectStore ${ }^{\circledR}$ propiciou a criação das estruturas de informações necessárias para representar o modelo de produto e Modelo de Produto Variante, o Visual $\mathrm{C}++\circledR$ propiciou o suporte para implementação das funcionalidades do sistema e geração de interfaces com o usuário.

Apesar de um conjunto de diferentes funções e soluções de projetos terem sido implementadas, este artigo mostrará um exemplo particular, relacionado com a alimentação do molde para explorar as idéias apresentadas no artigo.

A Figura 10 apresenta um ambiente geral do sistema construído, onde são mostradas três janelas: seleção das funções do molde; soluções de projeto associadas a uma função específica sem levar em consideração qualquer restrição de projeto; e especificações de projeto e decisões tomadas (sobreposta).
Após ser selecionada uma função específica (neste caso distribuir o galho de alimentação do molde Distribute Runner Layout) será apresentado um conjunto de soluções que, potencialmente, poderão atender a tal função. Neste caso, as soluções serão avaliadas em relação a suas interações, para verificar quais, deste conjunto de possíveis soluções, poderão ser utilizadas para o caso em questão.

Contudo, quando avaliadas com base em algumas especificações de projeto, armazenadas no modelo de produto, este conjunto de soluções divide-se em aceitas e rejeitadas. A Figura 11 mostra como o conjunto inicial de soluções potenciais ficou dividido entre soluções aceitas e rejeitadas após uma avaliação de suas interações com as especificações de projeto definidas, i.e. número de impressões (Number Impressions) com 4 impressões; configuração do molde (Mould Configuration) do tipo 2-placas; e tipo de alimentação (Runner Type) como fria. Isto é devido às interações associadas com cada uma destas soluções de projeto.

As interações associadas a cada solução de projeto poderão ser consideradas aprovadas ou reprovadas quando comparadas com as informações contidas no

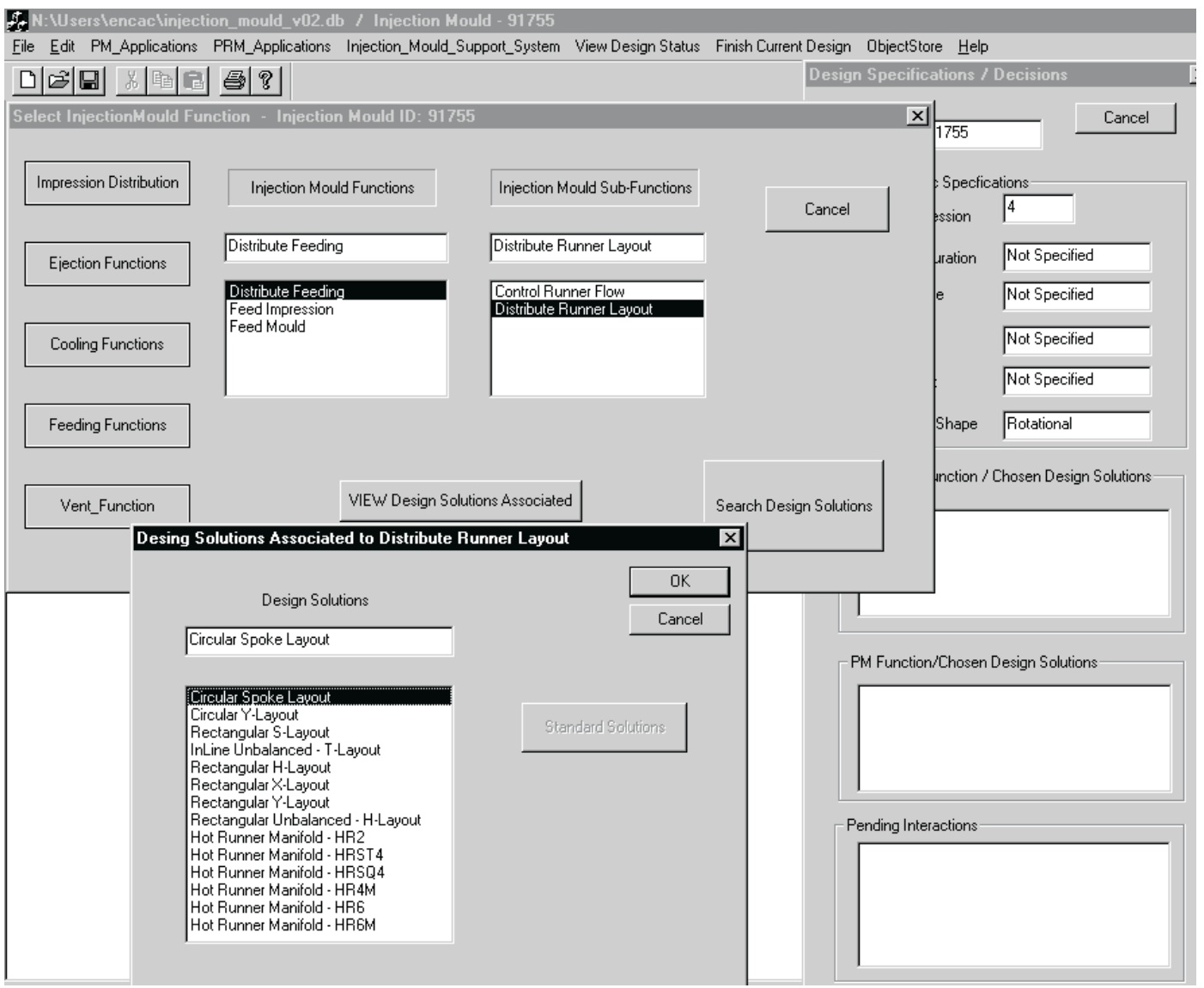

Figura 10 - Exemplo das solução de projeto associadas com uma função específica de alimentação 
modelo de produto. Na Figura 12 é mostrado que o conjunto de interações associadas a uma solução de projeto específica foi considerado aprovado. Contudo, uma das interações não pode ser avaliada em função da ausência da informação no modelo de produto, i.e. a forma como as impressões estão distribuídas no molde. Estes tipos de interações que não podem ser avaliadas, por si só não rejeitam uma solução de projeto, mas a colocam em estado de pendência. confirmar que a distribuição das 4 impressões no molde seja em linha (Distribution = In Line $)$. Tão logo tal definição seja feita, esta pendência desaparecerá. A existência de interações pendentes não impedirá que o projetista faça futuras escolhas de outras soluções de projetos, para atender outras funções do molde de injeção, desde que tal escolha não entre em conflito com estas pendências. Contudo, quanto mais especificadas e definidas as condições iniciais de

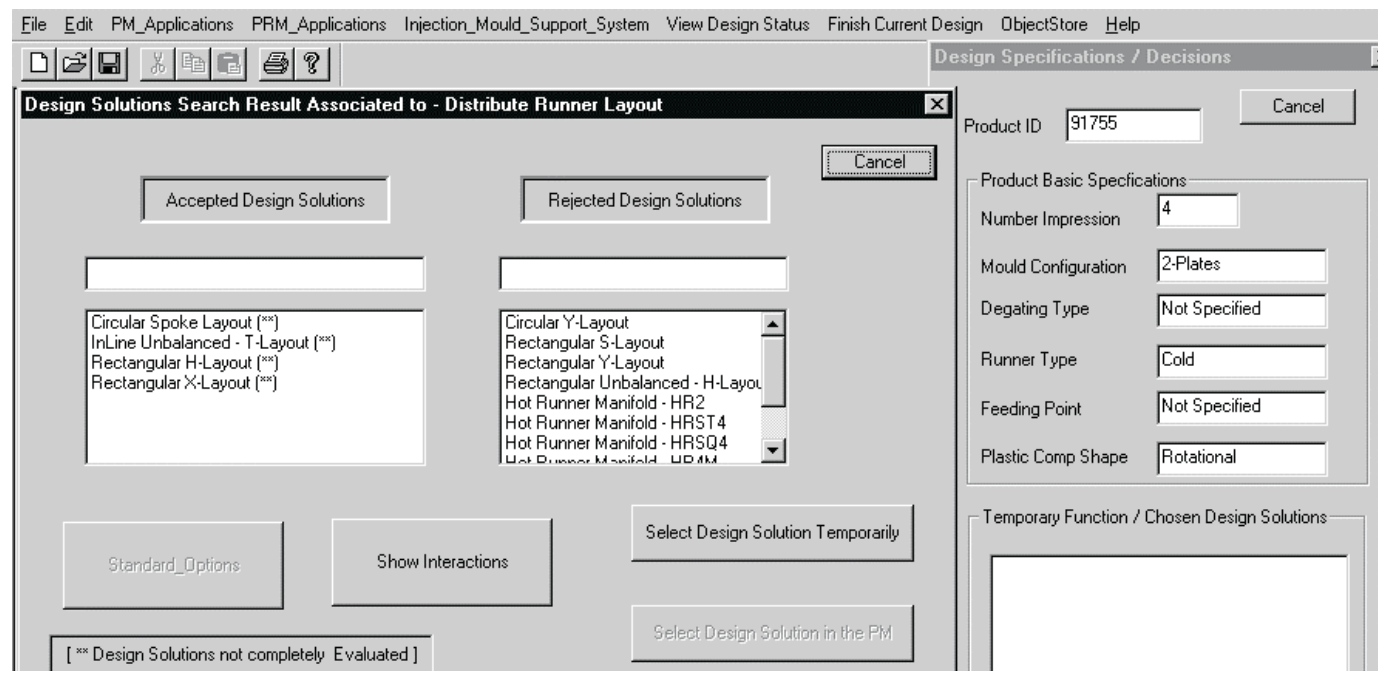

Figura 11 - Representação das soluções válidas para uma determinada especificação inicial de projeto

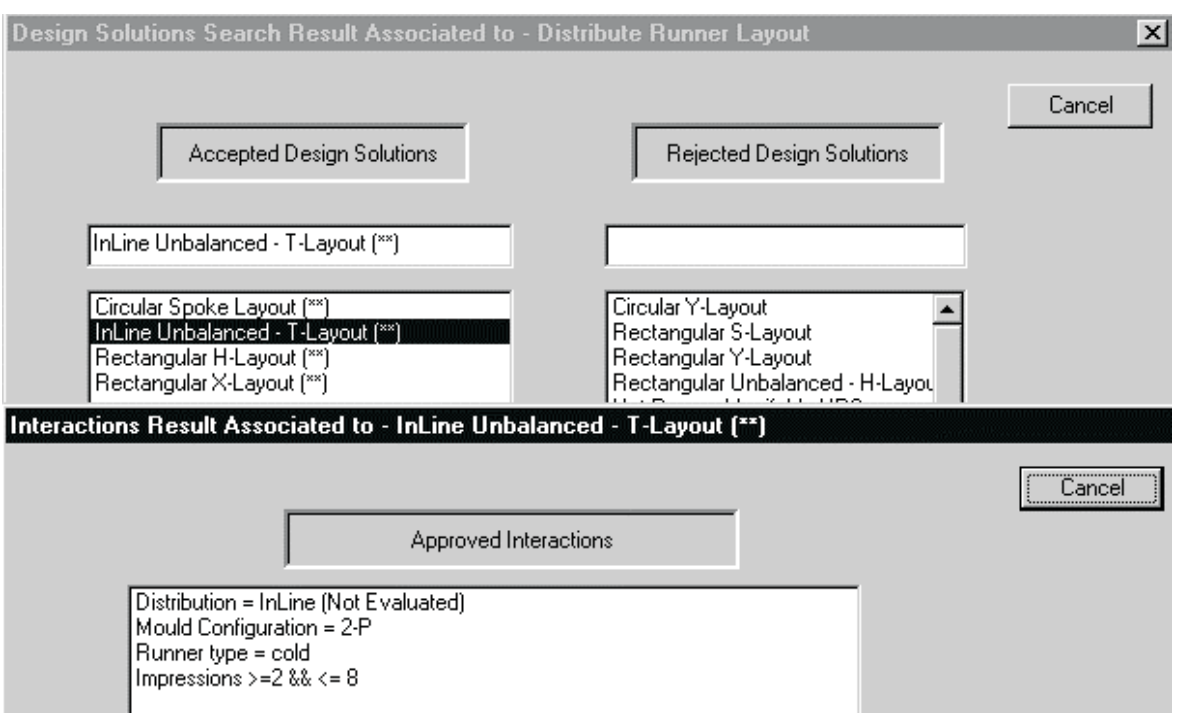

Figura 12 - Interações aprovadas de uma solução de projeto

A Figura 13 mostra que, após a seleção de uma solução de projeto, que tenha sido considerada aceita, contudo, com uma interação não avaliada, tal interação aparecerá como pendente, aguardando uma decisão do projetista. Neste caso, é mostrado que com a escolha da solução de layout para alimentação do tipo "T" (InLine Unbalanced - T Layout), existe a pendência de se projeto, menos interações deste tipo existirão. Outras pendências de interações poderão ocorrer entre diferentes soluções de projeto escolhidas para atender diferentes funções do molde, contudo esta é uma das maiores vantagens desta aborgem, ou seja, fornecer ao projetista uma boa orientação e flexibilidade na escolha das melhores opções de projeto. 


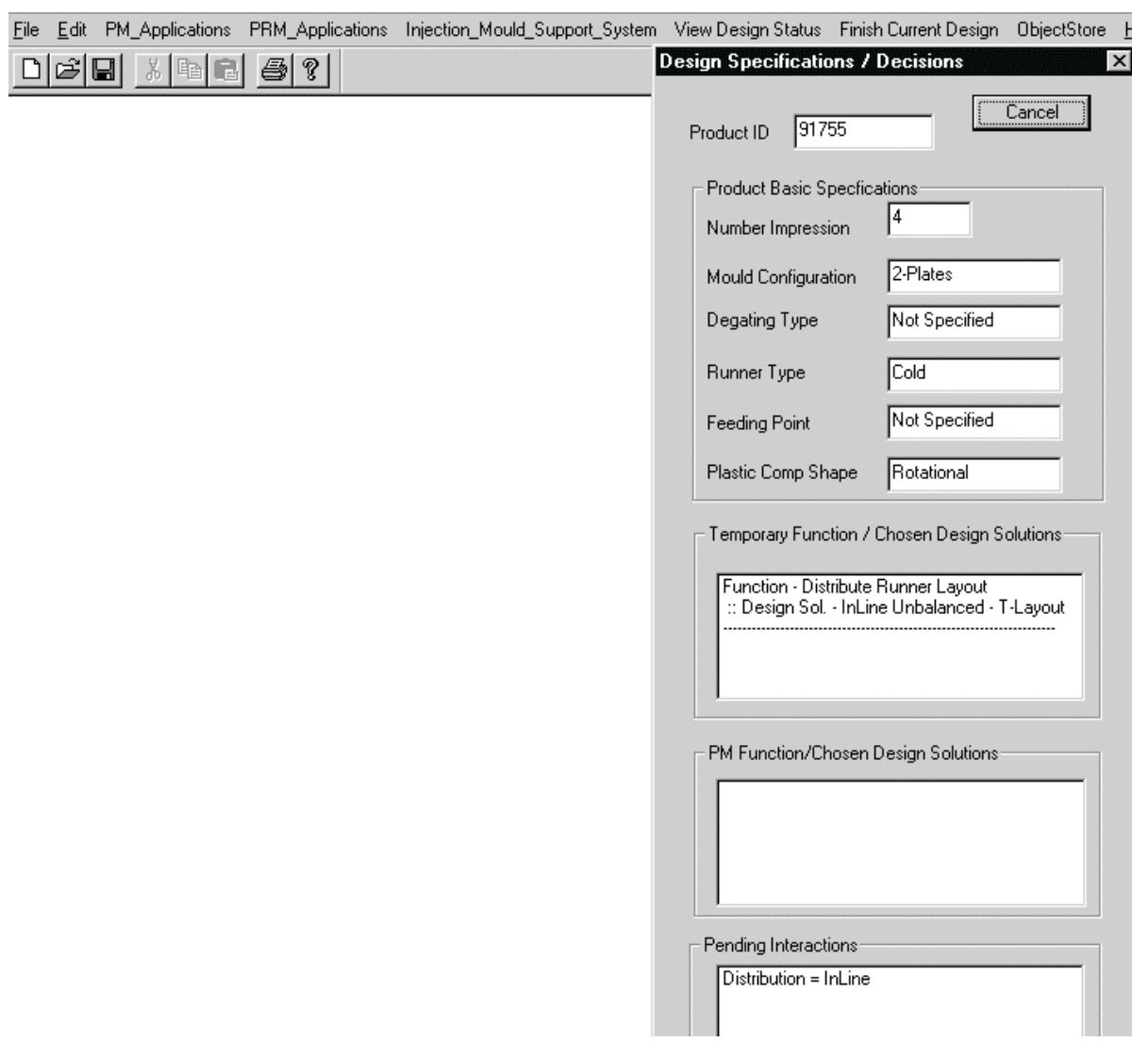

Figura 13 - Interação pendente associada a uma solução de projeto selecionada.

\section{Conclusão}

Este artigo discutiu o uso de estruturas de modelos de informações para o apoio à reutilização de informações no projeto. Neste, um novo tipo de modelo de informações, chamado Modelo de Produto Variante, foi proposto para apoiar a reutilização de informações no caso de projetos variantes. A estrutura geral deste modelo foi discutida e definida em termos de: funções e suas relações com possíveis soluções de projeto; as interações que estão associadas a cada uma destas potenciais soluções de projetos; e Links de Conhecimento que associam as interações com informações armazenadas no modelo de produto.

Apesar de se tratar de um modelo de informações separado do modelo de produto, o Modelo de Produto Variante mantém uma relação bastante próxima do modelo de produto quanto à estrutura dos dados. Por esta razão, é relevante que o desenvolvimento destes tipos de modelos de informações adicionais possuam seu desenvolvimento em paralelo a outros modelos de informações. Neste trabalho, a compatibilidade das estruturas entre os modelos foi avaliada em um nível mais superficial, sendo necessário um maior estudo caso esta compatibilidade se dê em um nível mais detalhado.
A abordagem utilizada permite que cada solução de projeto seja definida como um objeto individual que possui seu próprio conhecimento e relações definidas com as informações contidas no modelo de produto. Isto proporciona uma evolução do conhecimento heurístico adquirido ao longo do tempo, sendo possível sua atualização e amadurecimento à medida que novas idéias de projeto tornam-se aceitas. Contudo, maiores investigações necessitam ser feitas, com relação a como gerenciar este processo de evolução. Da mesma forma que as soluções de projeto, as interações foram definidas como objetos individuais e, como tais, podem ser reutilizadas para definirem diferentes soluções de projeto.

Por último, este trabalho mostrou como o conceito do Modelo de Produto Variante pode ser utilizado para oferecer às empresas uma habilidade de reutilizar suas informações e conhecimento para apoiar as equipes de projeto em suas tomadas de decisões.

\section{Referências}

ANDERL, R. Trends in Product Modelling. In: 11th International Conference on Engineering Design ICED’97, Tampere - Finland, August, 1997. p. 113-120 (Tampere University of Technology). 
CHIN, K.S.; WONG, T.N. Knowledge-based

Evaluation for the Conceptual Design Development of Injection Molding Parts. Engineering Applications in Artificial Intelligence, 1996. 9(4): p. 359-376.

COSTA, C.A.; YOUNG, R.I.M., Uma revisão em Sistemas Baseados em Inteligência Artificial para Suporte ao Projeto de Moldes de Injeção. Revista do CCET, Vol. 2, No. 2, 1999, pp. 14-32, ISSN 1516-4829

COSTA, C.A.; YOUNG, R.I.M. A Combined Knowledge and Information Infrastructure to Support Product Development. E-Business and Virtual Enterprises: Managing business-to-business cooperation, Ed. Camarinha-Matos, L.M., Afsarmanesh, H. and Rabelo, R.J., Kluwer Academic Publishers, 2001, p. 507-514.

COSTA, C.A.; HARDING, J.A.; YOUNG, R.I.M., The Application of UML and na Open Distributed Process Framework to Information System Design. Computers in Industry, Vol. 46:(1), 2001, pp. 19-36

ERENS, F.; VERHULST, K. Architectures for product families. Computers in Industry, 1997. 33: p. 165-178.

GU, P.; CHAN, K. Product Modelling using STEP. Computer-Aided Design, 1995. 27(3): p. 163-179.

HSU, W.; WOON, I.M.Y. Current Research in the Conceptual Design of Mechanical Products. Computer Aided Design, Vol. 30, No. 5, 1998, pp. 377-389.

IRANI, R.K.; KIM, B.H.; DIXON, J.R. Towards Automated Design of Feed System of Injection Molds by Integrating CAE, Iterative Redesign and Features. Transactions of the ASME, 1995. 117(February):p. 72-77.

JO, H.; PARSAEI, H.; SULLIVAN, W. Principles of concurrent engineering. In: Concurrent Engineering: Conteporary issues and modern design tools. Editores Parsaei, H.R. eSullivan, W.G. 1993, Chapman \& Hall: Cambridge.p. 3-23.

LEE, R.S.; CHEN, Y.M.; LEE, C.Z. Development of a concurrent mold design system: a knowledge-based approach. Computer Integrated Manufacturing Systems, 1997. 10(4): p. 287-307.

MANNISTO, T.; PELTONEN, H.; MARTIO, A.; SULONEN, R. Modelling generic product structures in STEP. Computer-Aided Design, 1998.30(14): p. 1111-1118.
MCKAY, A.; BLOOR, M.S.; DE PENNINGTON, A. A Framework for Product Data. IEEE Transactions on Knowledge and Data Engineering, 1996. 8(5): p. 825-837.

MENGES, G.; MOHREN, P. How to make injection molds, ed. 2. Carl Hanser Verlag, 1993.

MOLINA, A.; ELLIS, T.I.A.; YOUNG, R.I.M.; BELL, R. Modelling Manufacturing Capability to Support Concurrent Engineering. Concurrent Engineering: Research and Applications, 1995. 3(1): p. 29-42.

ONG, S.K.; PROMBANPONG, S.; LEE, K.S. An object-oriented approach to computer-aided design of a plastic injection mould. Journal of Intelligent Manufacturing, 1995. 6: p. 1-10.

ROSATO, D.V.; ROSATO, D.V. Injection Molding Handbook: The Complete Molding Operation Technology, Performance, Economics. 1995, New York: Chapman \& Hall.

SIVALOGANATHAN, S.; SHAHIN, T.M.M. Design reuse: on overview. Proc. Instn. Mech. Engrs, Part B, Journal of Engineering Manufacture, 1999. 213: p. 641-654.

WANG, Z.; LEE, K.S.; FUH, J.Y.H.; LI, Z.; ZHANG, Y.F.; NEE, A.Y.C.; YANG, D.C.H. Optimum ejector system design for plastic injection moulds. International Journal of Computer Applications in Technology, 1996. 9(4): p. 211-218.

Submetido em abril/2003

Aprovado em setembro/2004

\section{Agradecimentos}

Os autores agradecem ao $\mathrm{CNPq}$ (Conselho Nacional de Desenvolvimento Científico e Tecnológico) pela bolsa de doutorado concedida, à Universidade de Caxias do Sul pelo apoio financeiro e à FAPERGS (Fundação de Amparo a Pesquisa do Rio Grande do Sul) por propiciar condições para que a pesquisa possa ser continuada no Brasil. Agradecem também ao corpo de revisores das Revista Produto \& Produção por suas sugestões e contribuições para melhoria do artigo. 